\title{
Multifunctional Alginate Hydrogel Protects And Heals Skin Defects In Complex Clinical Situations
}

Wei Lu ${ }^{1 * \#}$, Dongyan Bao ${ }^{2 \#}$, Fangxin Ta ${ }^{3}$, Danping Liu ${ }^{1}$, Dezhi Zhang ${ }^{1}$, Zheng Zhang ${ }^{1}$, Zhongkai Fan ${ }^{*}$

1 Department of Orthopaedic surgery, The First Affiliated Hospital of Jinzhou Medical University,

Liaoning 121001, PR China

2Basic Medical College, Jinzhou Medical University, Liaoning 121001, PR China

3Health Management Center, The First Affiliated Hospital of Jinzhou Medical University,

Liaoning 121001, PR China

\#Wei Lu and Dongyan Bao are co-first authors of this article

*Corresponding author:

Zhongkai Fan( E-mail :flanzz@163.com)

Address: Department of Orthopaedic surgery, The First Affiliated Hospital of Jinzhou Medical University, Liaoning 121001, PR China

Te1:+86-0416-4197673 
The gelation time of various SAP hydrogels were tested by vialtilting method. ${ }^{1,} 2$ Briefly, different concentration of $\mathrm{AA}$ and PEI solution with or without $\mathrm{Sr}^{2+}$ were blended until the time that the solutions turn to globule was determined as the gelation time.

Table S1. Hydrogel preparation with various formulations.

\begin{tabular}{|c|c|c|c|c|}
\hline & PEI (10\%) & $\begin{array}{l}\mathrm{PEI} \\
\mathrm{Sr}^{2+}\end{array}$ & $\begin{array}{l}(10 \%) \& \\
(1 \mathrm{mg} / \mathrm{mL})\end{array}$ & $\begin{array}{l}\text { sodium alginate } \\
\text { aldehyde (10\%) }\end{array}$ \\
\hline $\mathrm{SA} 1$ & 1 & & & 1 \\
\hline $\mathrm{SA} 2$ & 1 & & & 2 \\
\hline SAP1 & & & 1 & 1 \\
\hline SAP2 & & & 1 & 2 \\
\hline
\end{tabular}

1. Strehin, I. ; Nahas, Z. ; Arora, K. ; Nguyen, T. ; Elisseeff, J., A versatile $\mathrm{pH}$ sensitive chondroitin sulfate-PEG tissue adhesive and hydroge1. Biomaterials 2010, 31 (10), 2788-97. 
2. Zhang, Z. ; Wang, X. ; Wang, Y. ; Hao, J., Rapid-Forming and SelfHealing Agarose-Based Hydrogels for Tissue Adhesives and Potential Wound Dressings. Biomacromolecules 2018, 19 (3), 980-988. 